\title{
AN AUTOMATIC REVERBERATION METER FOR THE MEASUREMENT OF SOUND ABSORPTION
}

\author{
By W. F. Snyder
}

\section{ABSTRACT}

The usual electrical recording method for measuring sound absorption in a reverberation room requires the determination of the rate of decay of sound. The automatic reverberation meter described in this paper works through a range of about 70 decibels, the rate of decay being measured over intervals as short as desired through the whole range.

An automatic control of the apparatus has been developed which allows measurements to be made with but little attention on the part of the observer.

The working ranges of the four methods ${ }^{1}$ that have been used at the Bureau of Standards for measuring the rate of decay of sound in a room are shown diagrammatically in Figure 1. The approximate

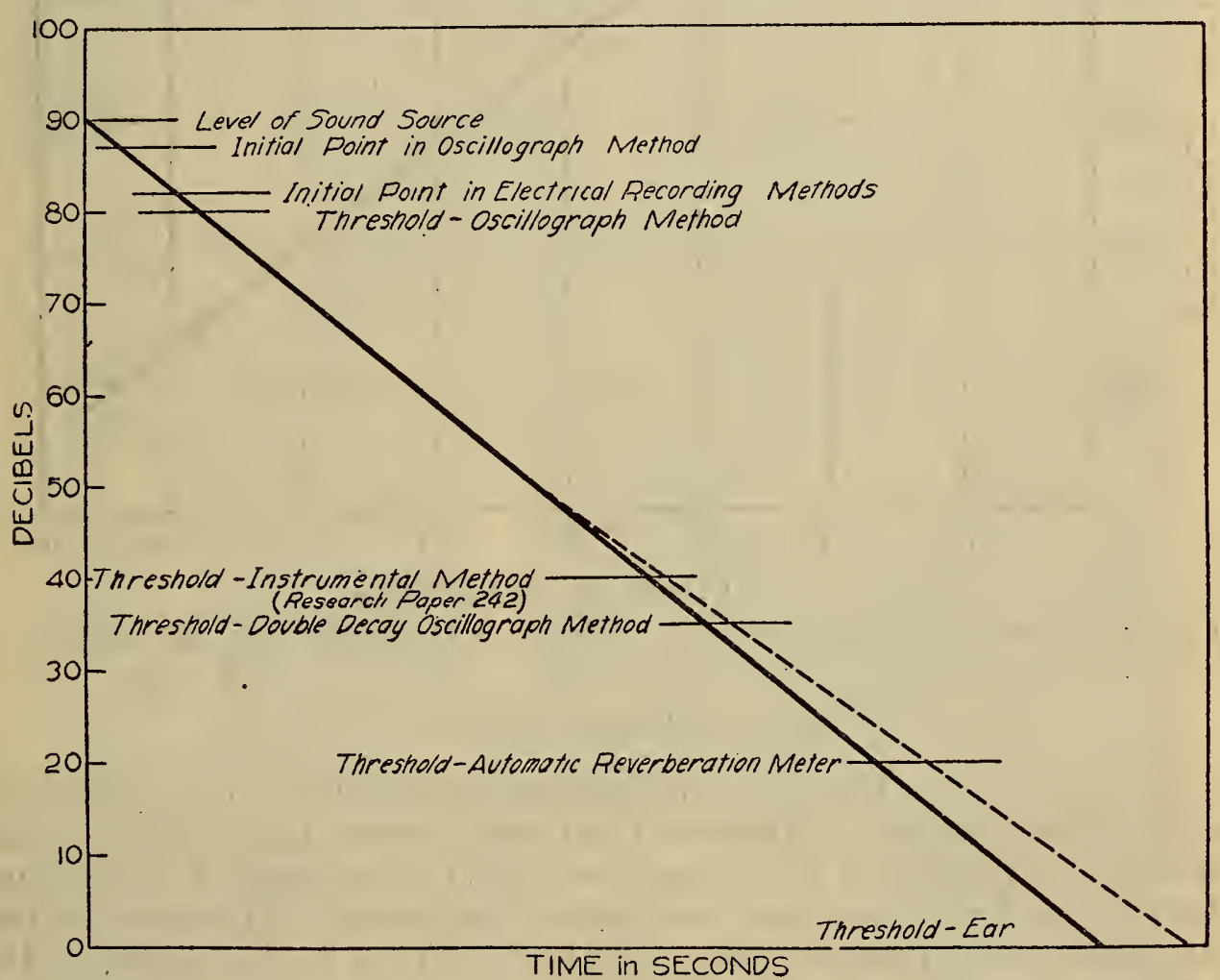

FIGURe 1.-Working ranges of various methods used at the Bureau of Standards to measure reverberation time

range through which the rate of decay is measured by each method is indicated by the difference in decibels between the initial point and the final threshold. The ordinary oscillograph method is very limited in range, being about 7 decibels. The other methods described were

\footnotetext{
1 See reference 6 in the bibliography.
}

$$
122486-32-4
$$


used through ranges of 40 to 50 decibels. The present automatic reverberation meter works through a range of about 70 decibels. The ear, of course, covers even a greater range since in reverberation measurements the ear must start at the initial sound level in the room and must follow the decay to the threshold of hearing.

More important, however, than a measurement over a wide range of intensity, is the accurate determination of the rate of decay of the sound energy throughout the measured range. Such a procedure is essential when measuring highly absorbent materials in a reverberation room or whenever "two-room" conditions exist." Under these conditions a double rate of decay may exist as shown by the dotted

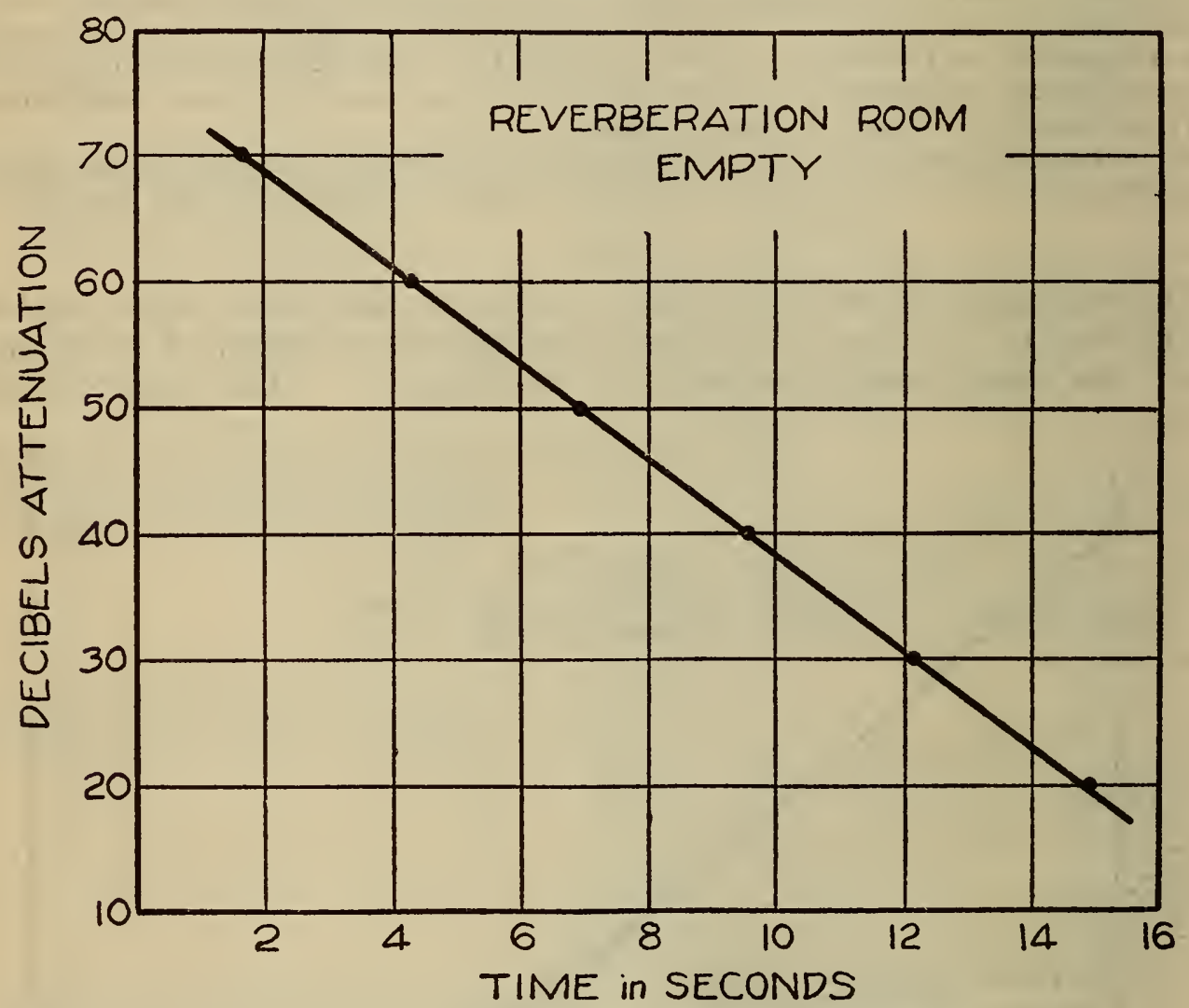

FIGURE 2.-Curve showing the rate of decay at 512 cycles made over a range of 50 decibels

It is the result of 60 observations.

line in Figure 1. The ear and instrumental methods previously described give but two points on the decay curve, the initial starting point and the threshold point, and we may be unaware of any change in the rate of decay between the points measured. The same is true in the double-decay oscillograph method. There is, however, in this case a short range at both the initial and threshold points where the rate is accurately known. The two short decay curves photographed on the oscillograms sometimes showed different rates of decay, indicating that the rate was not always uniform through the whole range.

Measurements with a reverberation meter as shown in Figure 2 yield a number of points along the decay curve at definite intervals, and the rate of decay may be determined throughout the working range. 


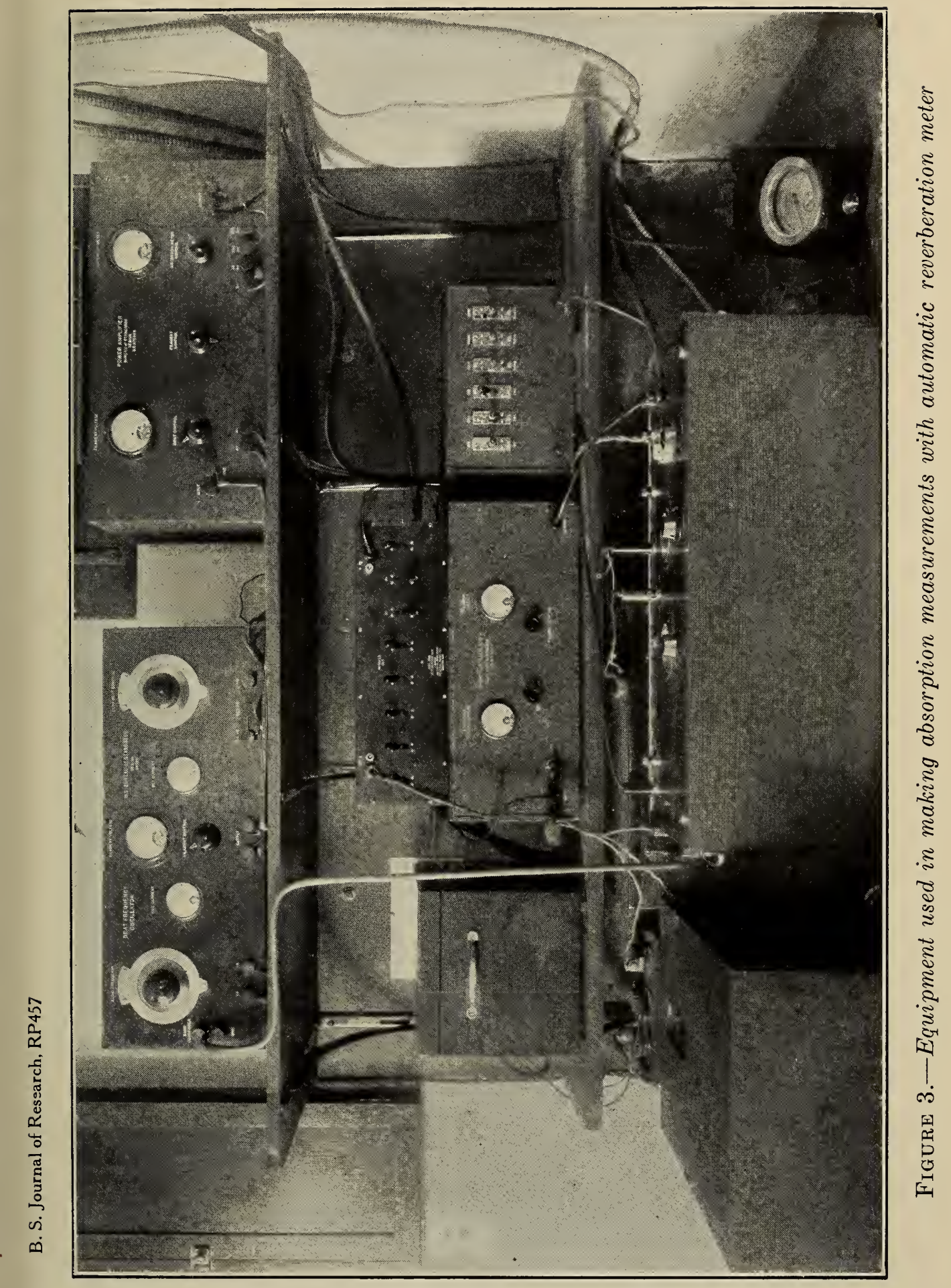


Since the decay of sound in a room is of a nonuniform character we must resort to a statistical method of measurement, each measurement being repeated at least ten times. The procedure in making these measurements is as follows: Sound radiated from a source such as a loud speaker is suddenly cut off and a timing mechanism started at the same time. The sound as it dies away is picked up by a microphone and when the sound reaches a certain level in the room an electrical arrangement stops the timer. The timer has then recorded the length of time the sound takes to decay from the initial intensity to the threshold at which the instrument is set. Shifting the threshold of the recording instrument by a definite number of decibels, a different time is measured. Knowing the time it takes the sound to decay through definite ranges of sound levels, a curve can be plotted similar to Figure 2 in which the slope gives the rate of decay. From the rate of decay the absorption of the room can be calculated by the reverberation equation. ${ }^{3}$

Making statistical measurements in such fashion becomes quite laborious. The present design of the reverberation meter used at the Bureau of Standards has made much of the operation automatic.

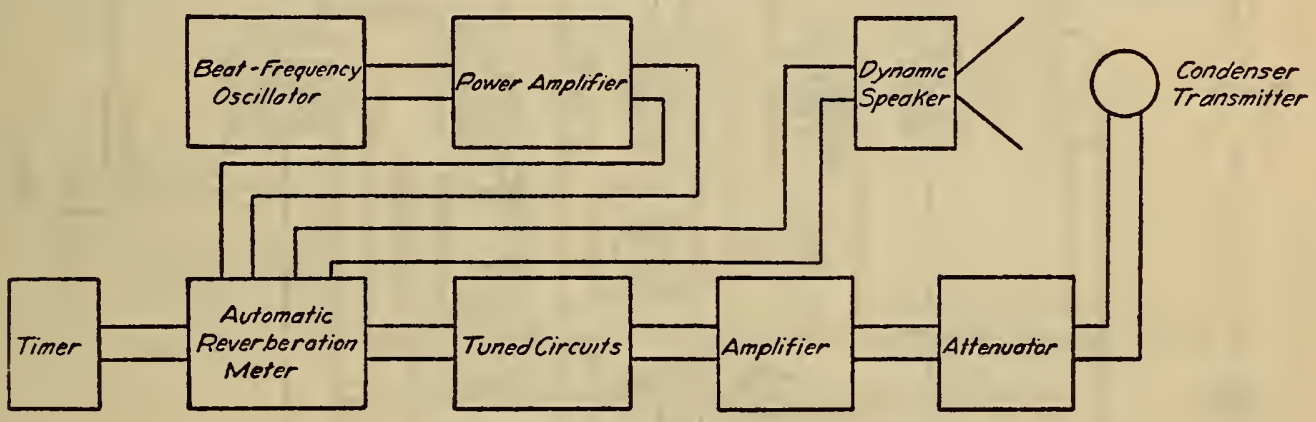

FIGURE 4.- Schematic diagram of entire apparatus

Figure 3 shows the recording equipment and Figure 4 is a schematic diagram of the entire apparatus. Alternating current in the audiofrequency range is generated by a beat-frequency oscillator, strengthened by a power amplifier and finally transformed into sound energy by a dynamic speaker. A condenser transmitter transforms the sound back into electrical energy. The weak electrical current is then amplified; the threshold of the instrument being controlled by an attenuator. Immediately following the amplifier is a group of tuned circuits which aid in reducing the level of extraneous noises. These tuned circuits attenuate approximately 15 decibels at the first octave above and below the frequency to which they are tuned. The automatic reverberation meter with its associated timer terminates the recording apparatus.

Figure 5 shows in detail the electrical circuit contained within the reverberation meter which controls the timer. There is one stage of amplification transformer coupled to a screen-grid tube. The screengrid tube which acts as a rectifier is directly coupled through a 0.5 megohm resistor to a pentode tube. The pentode tube is used as an oscillator and was so chosen because of its high plate current when connected in series with a high resistance relay. Some difficulty has been found in getting a pentode tube to oscillate under the particular

' See reference 6 in the bibliography. 
circuit requirements which are necessary for the desired operation of the equipment. The frequency of oscillation is about $700 \mathrm{kc}$ per second.

The grid bias of the pentode tube depends upon the potential across the plate resistor of the rectifier tube which in turn is a function of the sound energy picked up by the microphone. When the sound level is high, as at the beginning of the decay, the grid bias on the oscillator is sufficiently negative to prevent it from oscillating and the plate current is nearly zero. As the sound dies away the grid bias becomes more positive. This continues until a sufficiently positive bias sets the tube into oscillation with a sudden increase in plate current. In consequence, a relay in series with the tube opens a set of contacts, stopping the timer. The sudden increase in current

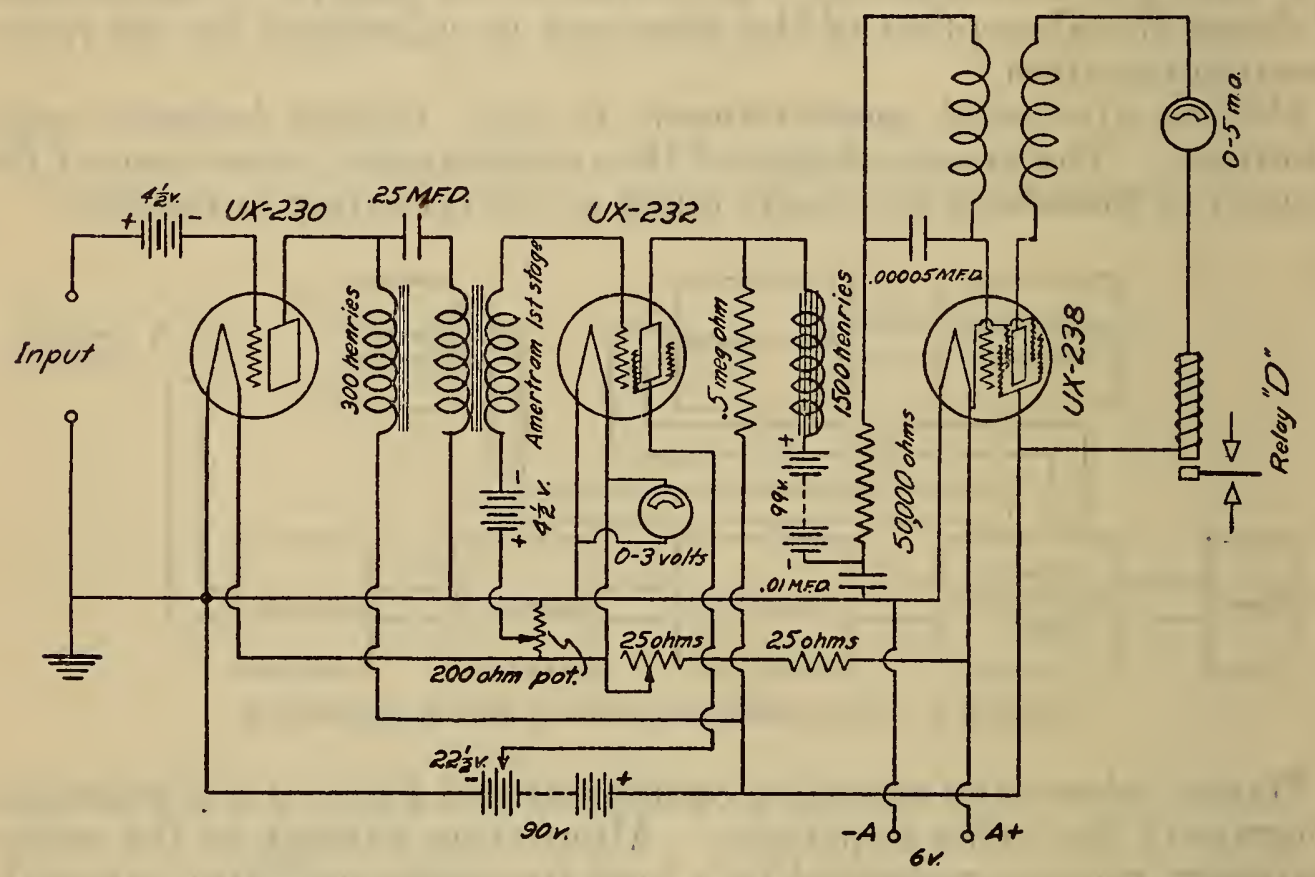

Figure 5.-Electrical circuit diagram of apparatus which stops the electric timer

through the relay winding gives a positive control to the operation. The point at which this goes into operation, which has been called the threshold of the instrument, depends upon the amount of amplification used and the setting of the attenuator.

The 1,500-henry choke in series with the grid circuit of the pentode tube and the $0.01 \mu \mathrm{f}$ by-pass condenser serve as a filter for the audio frequencies. This is a necessary precaution to prevent the relay from humming when low frequencies are measured. The circuit is so designed that once the tube goes into oscillation a negative shift in the grid bias caused by an increase in the sound energy amounting to about 4 decibels is necessary to stop oscillations, thereby restarting the timer. This more or less helps to integrate out a variable sound decay and at the same time prevents the timer from following variations less than 4 decibels which would cause excessive starting and stopping of the timer.

Figure 6 shows the arrangement of relays which makes the operation of the reverberation meter almost automatic. The apparatus con- 
sists of three relays, a small alternating current motor with a 1,100 to 1 reduction gear coupled by a shaft to a cam operating a 4-pole switch and a self-stopping device. Large condensers across the contacts of the relays prevent sparking and setting up electrical disturbances in the near-by amplifying equipment.

The operation of this device is as follows: A rod connected to the armature of relay $A$ is lifted, closing the motor circuit. When no sound is on, the lower contact at relay $D$ (fig. 6) is closed, allowing current to flow through the windings of relay $A$, thus holding the armature of relay $A$ after it is lifted.

Connected to the same armature is a small pin fitting into cam $B$, which when lifted allows relay $A$ to operate properly, since the armature is now close to the pole piece, the pin riding around on top of cam $B$. A 4-pole switch operated by the segments along cam $A$ is closed after the cam begins to rotate. This switch closes the loud-speaker circuit and closes a circuit through the winding of relay $A$ which

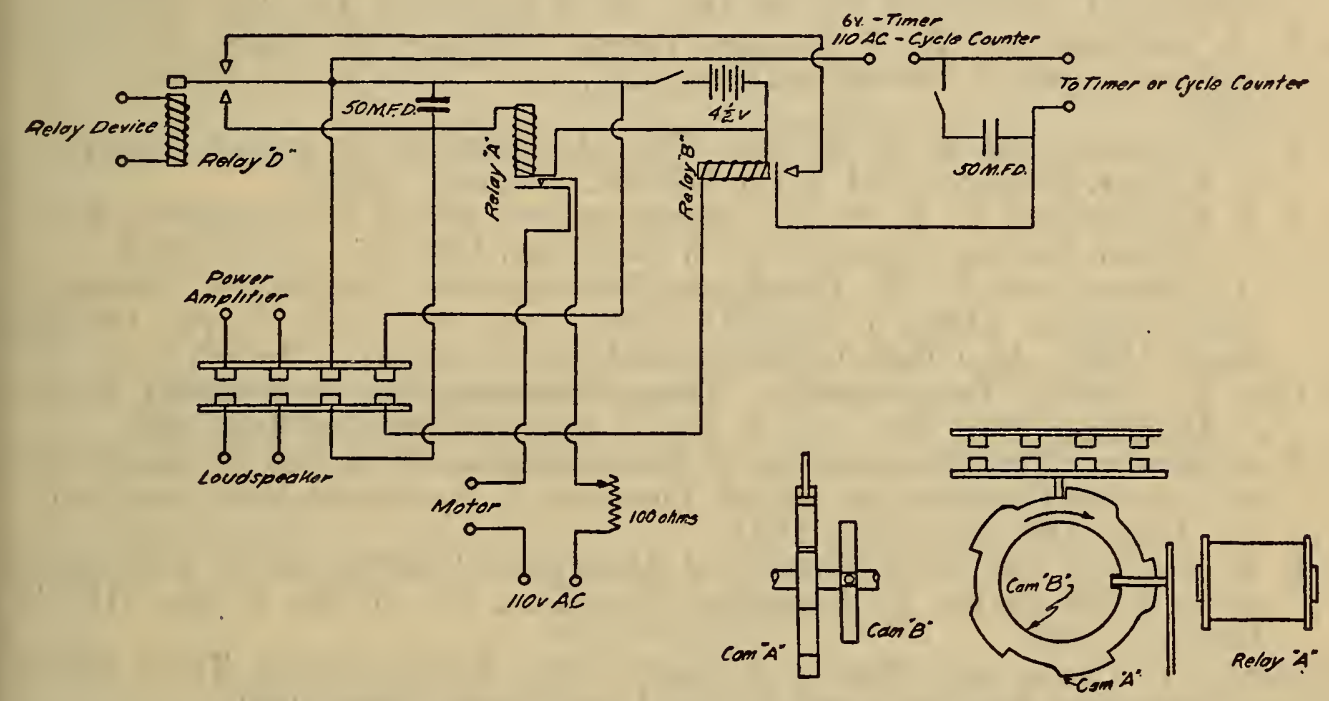

FIGURE 6.-Relays and cams which provide automatic control for the reverberation meter

normally opens at relay $D$ as the sound comes on. It also closes the circuit of the winding of relay $B$ which prevents the timer from operating. After a further rotation of the cam shaft the 4-pole switch suddenly springs open breaking the loud-speaker circuit, opening the motor circuit and allowing the contact of relay $B$ to close, which starts the timer at the beginning of the decay. While the sound is on or as it is decaying the timer contact of relay $D$ is closed. As the sound decays to the threshold of the instrument and the plate current suddenly increases in the pentode tube, relay $D$ operates stopping the timer. At the same time the other contact on relay $D$ closes and the motor is started. The whole process is repeated. This continues for five measurements. At the fifth measurement the pin on the armature of relay $A$ drops back into the slot of cam $B$ and stops the entire mechanism.

An average of 10 observations is usually taken at six different sound levels spaced at 10 decibel intervals making a range of 50 decibels. This is sufficient for most absorption measurements. The apparatus has a range, however, of about 70 decibels. 
The apparatus described above offers a statistical method of dealing with a type of measurement that presents certain difficulties, since we are determining a rate of decay that is seldom uniform. The method averages a series of observations in a mechanical fashion and is almost entirely automatic. This being true, the human equation in reverberation and absorption measurements has been practically eliminated.

\section{BIBLIOGRAPHY OF PUBLISHED ARTICLES REFERRING TO ME- CHANICAL AND ELECTRICAL METHODS OF MEASURING REVER- BERATION TIME}

1. E. Meyer and Paul Just, On the Measurement of Reverberation Time and Sound Absorption, Elek. Nach. Tech., vol. 5, pp. 293-300, 1928.

2. E. Meyer, Automatic Reverberation Measurement, Zeit. f. Tech. Physik., vol. 11 , pp. 253-259, 1930.

3. M. J. O. Strutt, Automatic Reverberation Measuring Instrument, Elek. Nach. Tech., vol. 7, pp. 280-282, July, 1930.

4. V. L. Chrisler, The Measurement of Sound Absorption by Oscillograph Records, J. Acoustical Soc. of Am., vol. 1, No. 3, pp. 418-421, April, 1930.

5. V. L. Chrisler and W. F. Snyder, Recent Advances in Sound Absorption Measurements, J. Acoustical Soc. Am., vol. 2, No. 1, pp. 123-128, July, 1930.

6. V. L. Chrisler and W. F. Snyder, The Measurement of Sound Absorption, B. S. Jour. Research, vol. 5, pp. 957-972, October, 1930.

7. V. L. Chrisler and W. F. Snyder, Measurements with a Reverberation Meter, J. Motion Picture Engrs., vol. 18, No. 4, pp. 479-487. April, 1932.

8. E. C. Wente and E. H. Bedell, A Chronographic Method of Measuring Reverberation Time, J. Acoustical Soc. Am., vol. 1, No. 3, pp. 422-427, April, 1930. Also Bell Telephone Laboratory Reprint B-500.

9. Carl F. Eyring, Reverberation Time Measurements in Coupled Rooms, J. Acoustical Soc. Am., vol. 3, No. 2, pp. 181-206, October, 1930.

10. F. L. Hopper, The Measurement of Reverberation Time and Its Application to Acoustic Problems in Sound Pictures, J. Acoustical Soc. Am., vol. 2, No. 4, pp. 499-505, April, 1931.

11. F. L. Hopper, The Determination of Absorption Coefficients for Frequencies up to 8,000 cycles, J. Acoustical Soc. Am., vol. 3, No. 3, pp. 415-427, January, 1932.

12. Harry F. Olson and Barton Kreuzer, The Reverberation Time Bridge, J. Acoustical Soc. Am., vol. 2, No. 1, pp. 78-82, July, 1930.

13. R. F. Norris and C. A. Andree, An Instrumental Method of Reverberation Measurement, J. Acoustical Soc. Am., vol. 1, No. 3, pp. 366-372, April, 1930.

14. R. F. Norris, Application of Norris-Andree Merhod of Reverberation Measurement to Measurements of Sound Absorption, J. Acoustical Soc. Am., vol. 3, No. 3, pp. 361-370, January, 1932.

Washington, April 26, 1932. 